LAWRENCE LIVERMORE NATIONAL LABORATORY
High-Order Boundary Condition Perturbation Theory for the Neutron Transport Equation

M. S. McKinley and F. Rahnema

September 19, 2001 


\section{DISCLAIMER}

This document was prepared as an account of work sponsored by an agency of the United States Government. Neither the United States Government nor the University of California nor any of their employees, makes any warranty, express or implied, or assumes any legal liability or responsibility for the accuracy, completeness, or usefulness of any information, apparatus, product, or process disclosed, or represents that its use would not infringe privately owned rights. Reference herein to any specific commercial product, process, or service by trade name, trademark, manufacturer, or otherwise, does not necessarily constitute or imply its endorsement, recommendation, or favoring by the United States Government or the University of California. The views and opinions of authors expressed herein do not necessarily state or reflect those of the United States Government or the University of California, and shall not be used for advertising or product endorsement purposes.

This work was performed under the auspices of the U. S. Department of Energy by the University of California, Lawrence Livermore National Laboratory under Contract No. W-7405-Eng-48.

This report has been reproduced directly from the best available copy.

Available electronically at http://www.doc.gov/bridge

Available for a processing fee to U.S. Department of Energy

And its contractors in paper from

U.S. Department of Energy

Office of Scientific and Technical Information

P.O. Box 62

Oak Ridge, TN 37831-0062

Telephone: (865) 576-8401

Facsimile: (865) 576-5728

E-mail: reports@adonis.osti.gov

Available for the sale to the public from

U.S. Department of Commerce

National Technical Information Service

5285 Port Royal Road

Springfield, VA 22161

Telephone: (800) 553-6847

Facsimile: (703) 605-6900

E-mail: orders@ntis.fedworld.gov

Online ordering: http://www.ntis.gov/ordering.htm

OR

Lawrence Livermore National Laboratory

Technical Information Department's Digital Library

http:/ / www.llnl.gov/tid/Library.html 
September 19, 2001

\title{
HIGH-ORDER BOUNDARY CONDITION PERTURBATION THEORY FOR THE NEUTRON TRANSPORT EQUATION
}

\author{
Michael Scott McKinley and Farzad Rahnema \\ Georgia Institute of Technology \\ Nuclear Engineering and Health Physics Programs \\ The George W. Woodruff School of Mechanical Engineering \\ Atlanta, GA 30332-0405 USA \\ Email: mckinlev9@llnl.gov and farzad.rahnema@me.gatech.edu
}

Number of pages: 32 (including this page)

Number of Tables: 9

Number of Figures: 2

Send correspondences to:

Farzad Rahnema

Georgia Institute of Technology

Nuclear Engineering and Health Physics Programs

The George Woodruff School of Mechanical Engineering

Atlanta, GA 30332-0405

Voice: (404) 894-3731

Fax: (404) 894-3733

E-mail: $\quad$ farzad.rahnema@me.gatech.edu 


\title{
HIGH-ORDER BOUNDARY CONDITION PERTURBATION THEORY FOR THE NEUTRON TRANPSORT EQUATION
}

\author{
Michael Scott McKinley ${ }^{1}$ and Farzad Rahnema \\ Georgia Institute of Technology \\ Nuclear Engineering and Health Physics Programs \\ The George W. Woodruff School of Mechanical Engineering \\ Atlanta, GA 30332-0405 USA \\ Email:farzad.rahnema@me.gatech.edu
}

\begin{abstract}
First-order boundary condition perturbation theory is extended to $n^{\text {th }}$-order in transport theory for eigenvalue problems. In particular, using an unperturbed (known) solution, formalisms are developed to determine the solution to the neutron transport equation when the boundary condition of the system is perturbed. The new method requires the computation of an adjoint Green's function. The numerical solution of this function is discussed. Finally, four numerical examples are provided to verify the validity of the formalisms presented.
\end{abstract}

\section{INTRODUCTION}

Boundary condition perturbation was first introduced into reactor physics in $1977^{1}$ for simple idealized geometries in diffusion theory. In 1981, the theory was further extended by allowing for a 
first-order prediction of the eigenvalue for extrapolation distance in diffusion theory. ${ }^{2}$ However, the first-order eigenvalue solution was not explicitly given until 1989. ${ }^{3}$ In Ref. 3, ratios of linear functionals were given for a current-to-flux boundary condition perturbation which is useful in homogenization techniques in nodal methods. ${ }^{4}$ In 1994, Ref. 5 completes the first-order boundary perturbation theory in diffusion theory by solving for the first-order flux.

The first-order eigenvalue was not considered in transport theory until $1996 .{ }^{6}$ First-order change in linear functionals in transport theory was explored the following year. ${ }^{7}$ In 1998, a theory was developed in first-order to treat a boundary perturbation as a boundary condition perturbation. ${ }^{8}$ High-order boundary condition perturbation theory started as a solution to the second-order eigenvalue ${ }^{9}$ and then finally reached arbitrary order in the diffusion approximation. ${ }^{10}$ Reference 10 was used to develop a high-order cross section homogenization method to improve the coarse-mesh nodal accuracy in diffusion theory. ${ }^{11}$ The high-order boundary condition theory is needed to improve the accuracy of the discontinuity factor in equivalence theory ${ }^{12}$ since first-order approximation is not accurate enough.

The purpose of this paper is to present a formalism for a high-order boundary condition analysis in transport theory. The next section has a derivation of the first-order theory that has previously been discussed ${ }^{6,7}$ and continues the work to higher-order. In Sec. III, we show how numerical techniques may be applied to solve for the adjoint Green's function needed for the derivation of expansion coefficients. Four numerical example problems are given in Sec. IV to demonstrate the

\footnotetext{
${ }^{1}$ Current address: Lawrence Livermore National Laboratory, L-414 LLNL, Livermore, CA 94550, mckinley9@llnl.gov
} 
correctness of the theory. Section V discusses the propagation of the errors that limit the accuracy of the high-order method. The paper concluded in section VI.

\section{DERIVATION}

We begin our analysis by first defining a compact notation for the phase space integration:

$$
\begin{gathered}
<a b>\equiv \int_{\Re} d^{3} x \int_{E} d E \int_{4 \pi} d^{2} \Omega a b \text { and } \\
<a b>_{S} \equiv \int_{\delta \Re} d^{2} x \int_{E} d E \int_{4 \pi} d^{2} \Omega a b
\end{gathered}
$$

where $x$ is the physical dimensions, $E$ is the neutron energy, $\Omega$ is the angular direction, and $a$ and $b$ are arbitrary operators and/or functions. The surface integral in Eq. (2) may further be modified by specifying certain angular directions such as $\langle a b\rangle_{s, \hat{\Omega} \cdot \hat{n}<0}$, which limits the integral to only incoming directions.

The first step starts with the steady-state homogenous (eigenvalue) transport equation in a given domain $\Re$ for the unperturbed case:

$$
H \bar{\varphi}_{0}=\lambda_{0} F \bar{\varphi}_{0}
$$

where $\mathrm{H}$ and $\mathrm{F}$ are the transport operators defined as

$$
\begin{gathered}
H=\hat{\Omega} \cdot \nabla+\sigma(\vec{x}, E)-\int d E^{\prime} \int_{4 \pi} d^{2} \Omega^{\prime} \sigma_{s}\left(\vec{x} ; E^{\prime}, \hat{\Omega}^{\prime} \rightarrow E, \hat{\Omega}\right) \text { and } \\
F=\int d E^{\prime} \int_{4 \pi} d^{2} \Omega^{\prime} v\left(\vec{x}, E^{\prime}\right) \sigma_{f}\left(\vec{x} ; E^{\prime}, \hat{\Omega}^{\prime} \rightarrow E, \hat{\Omega}\right)
\end{gathered}
$$

Where, $\sigma$ is the total cross section, $\sigma_{s}$ is the scattering kernel, $v$ is the average number of neutrons released per fission, and $\sigma_{f}$ is the fission cross section. We define the assumed known, unperturbed 
normalized flux, $\bar{\varphi}_{o}(\vec{x}, E, \hat{\Omega})$, such that the phase space integration of it is exactly one. The initial eigenvalue, $\lambda_{0}$, is also assumed known and is defined as the inverse of the effective multiplication factor, $k_{\text {eff. }}$ Common to many reactor physics applications, we choose to write the boundary condition as specular reflection boundary condition, which may be expressed as:

$$
\bar{\varphi}_{0}(\vec{x}, E, \hat{\Omega})=\gamma_{0} \bar{\varphi}_{0}\left(\vec{x}, E, \hat{\Omega}_{r}\right), \vec{x} \in \partial \Re, \hat{\Omega} \cdot \hat{n}<0
$$

where $\hat{n}$ represents the outward unit normal direction to the boundary and $\hat{\Omega}_{r}$ is the unit vector in the direction of specular reflection at the surface. The vacuum boundary condition is obtained by setting $\gamma_{0}$ to zero.

Perturbing the boundary condition by a small amount yields the following perturbed equation:

$$
H \bar{\varphi}=\lambda F \bar{\varphi}
$$

where $\lambda$ is the perturbed eigenvalue and $\bar{\varphi}$ is the perturbed flux that is normalized so that its phase space integral is equal to one. We assume that we can expand the normalized flux and eigenvalue in powers of a smallness parameter, $\varepsilon$, as shown below:

$$
\begin{aligned}
& \lambda=\lambda_{0}+\varepsilon \lambda_{1}+\varepsilon^{2} \lambda_{2} \cdots+\varepsilon^{n} \lambda_{n}+O\left(\varepsilon^{n+1}\right) \\
& \bar{\varphi}=\bar{\varphi}_{0}+\varepsilon \bar{\varphi}_{1}+\varepsilon^{2} \bar{\varphi}_{2} \cdots+\varepsilon^{n} \bar{\varphi}_{n}+O\left(\varepsilon^{n+1}\right)
\end{aligned}
$$

Integrating Eq. (9) over the phase space and equating terms of powers of $\varepsilon$ gives the following condition:

$$
<\bar{\varphi}_{i}>=\left\{\begin{array}{l}
1, i=0 \\
0, i>0
\end{array}\right\}
$$

Substituting the expansions of the eigenvalue and flux from Eqs. (8) and (9) into Eq. (7) and combining terms with common powers of $\varepsilon$ results in a series of equations, the first equation that 
corresponds to $\varepsilon^{0}$ terms is the initial unperturbed equation, Eq. (3). The series of equations continue as

$$
\begin{gathered}
\left(H-\lambda_{0} F\right) \bar{\varphi}_{1}=\lambda_{1} F \bar{\varphi}_{0} \\
\left(H-\lambda_{0} F\right) \bar{\varphi}_{2}=F\left(\lambda_{1} \bar{\varphi}_{1}+\lambda_{2} \bar{\varphi}_{0}\right) \\
\vdots \\
\left(H-\lambda_{0} F\right) \bar{\varphi}_{n}=F\left(\lambda_{1} \bar{\varphi}_{n-1}+\lambda_{2} \bar{\varphi}_{n-2} \cdots+\lambda_{n} \bar{\varphi}_{0}\right)
\end{gathered}
$$

We assume there is a perturbation in the albedo that takes the form of

$$
\bar{\varphi}(x, E, \hat{\Omega})=\left(\gamma_{0}+\varepsilon \gamma_{1}\right) \bar{\varphi}\left(\vec{x}, E, \hat{\Omega}_{r}\right), \vec{x} \in \partial \Re, \hat{\Omega} \cdot \hat{n}<0 .
$$

Expanding the above equation with the expansions from Eqs. (8) and (9) and equating terms of powers of $\varepsilon$ gives another series of equations. The first equation reduces to the unperturbed boundary condition found in Eq. (6). The other formulas are listed in order of the powers of $\varepsilon$ :

$$
\begin{gathered}
\bar{\varphi}_{1}(\vec{x}, E, \hat{\Omega})=\gamma_{0} \bar{\varphi}_{1}\left(\vec{x}, E, \hat{\Omega}_{r}\right)+\gamma_{1} \bar{\varphi}_{0}\left(\vec{x}, E, \hat{\Omega}_{r}\right), \vec{x} \in \partial R, \hat{\Omega} \cdot \hat{n}<0 \\
\bar{\varphi}_{2}(\vec{x}, E, \hat{\Omega})=\gamma_{0} \bar{\varphi}_{2}\left(\vec{x}, E, \hat{\Omega}_{r}\right)+\gamma_{1} \bar{\varphi}_{1}\left(\vec{x}, E, \hat{\Omega}_{r}\right), \vec{x} \in \partial R, \hat{\Omega} \cdot \hat{n}<0 \\
\vdots \\
\bar{\varphi}_{n}(\vec{x}, E, \hat{\Omega})=\gamma_{0} \bar{\varphi}_{n}\left(\vec{x}, E, \hat{\Omega}_{r}\right)+\gamma_{1} \bar{\varphi}_{n-1}\left(\vec{x}, E, \hat{\Omega}_{r}\right), \vec{x} \in \partial R, \hat{\Omega} \cdot \hat{n}<0
\end{gathered}
$$

The above equations are also valid for the unnormalized flux as well.

With above expressions, we can now solve for the first-order eigenvalue. We begin by operating on Eq. (11) with $\left\langle\bar{\varphi}_{0}^{*}, \cdot>\right.$ where $\bar{\varphi}_{0}^{*}$ is a yet to be determined function. After applying several manipulations we obtain the following equation: 


$$
<\bar{\varphi}_{1} \bar{\varphi}_{0}^{*} \hat{\Omega} \cdot \hat{n}>_{s}+<\bar{\varphi}_{1}\left(H^{*}-\lambda_{0} F^{*}\right) \bar{\varphi}_{0}^{*}>=\lambda_{1}<\bar{\varphi}_{0}^{*} F \bar{\varphi}_{0}>
$$

where

$$
\begin{gathered}
H^{*} \equiv-\hat{\Omega} \cdot \nabla+\sigma(\vec{x}, E)-\int_{E^{\prime}} d E^{\prime} \int_{4 \pi} d^{2} \Omega^{\prime} \sigma_{S}\left(\vec{x} ; E, \hat{\Omega} \rightarrow E^{\prime}, \hat{\Omega}^{\prime}\right) \\
F^{*} \equiv v(\vec{x}, E) \int_{E^{\prime}} d E^{\prime} \int_{4 \pi} d^{2} \Omega^{\prime} \sigma_{f}\left(\vec{x} ; E, \hat{\Omega} \rightarrow E^{\prime}, \hat{\Omega}^{\prime}\right)
\end{gathered}
$$

Define $\bar{\varphi}_{0}^{*}$ as

$$
\begin{gathered}
\left(H^{*}-\lambda_{o} F^{*}\right) \bar{\varphi}_{o}^{*}=0, \vec{x} \in \Re \\
\bar{\varphi}_{0}^{*}(\vec{x}, E, \hat{\Omega})=\gamma_{0} \bar{\varphi}_{0}^{*}\left(\vec{x}, E, \hat{\Omega}_{r}\right), \vec{x} \in \partial \Re, \hat{\Omega} \cdot \hat{n}<0 .
\end{gathered}
$$

The surface integral term in Eq. (18) is simplified by making use of Eqs. (15) and (22) in the expression. This results in a surface integral term that is only integrated over the incoming directions. Since the second term in Eq. (18) drops out due to Eq. (21), we have an expression for the first-order eigenvalue as

$$
\lambda_{1}=\frac{<\gamma_{1} \bar{\varphi}_{0}^{*}(\hat{\Omega}) \bar{\varphi}_{0}\left(\hat{\Omega}_{r}\right) \hat{\Omega} \cdot \hat{n}>_{s, \hat{\Omega} \cdot \hat{n}<0}}{<\bar{\varphi}_{0}^{*} F \bar{\varphi}_{0}>}
$$

which is equivalent to Eq. (19) in Ref. 6.

To complete the first-order theory, we need to solve for the flux. We can write a relationship for the normalized and unnormalized flux with a Dirac delta function as

$$
\bar{\varphi}\left(\vec{r}_{0}\right)=\frac{<\delta\left(\vec{r}, \vec{r}_{0}\right) \varphi(\vec{r})>}{<\varphi(\vec{r})>}
$$

where $\vec{r}$ and $\vec{r}_{0}$ are phase space tensors. This relationship may be expanded as 


$$
\begin{aligned}
& \left(\bar{\varphi}_{0}+\varepsilon \bar{\varphi}_{1}+\varepsilon^{2} \bar{\varphi}_{2} \cdots \varepsilon^{n} \bar{\varphi}_{n}\right)\left(<\varphi_{0}>+\varepsilon<\varphi_{1}>+\varepsilon^{2}<\varphi_{2}>\cdots+\varepsilon^{n}<\varphi_{n}>\right) \\
& =<\delta, \varphi_{0}>+\varepsilon<\delta, \varphi_{1}>+\varepsilon^{2}<\delta, \varphi_{2}>\cdots+\varepsilon^{n}<\delta, \varphi_{n}>+O\left(\varepsilon^{n+1}\right)
\end{aligned} .
$$

Multiplying out and equating terms of $\varepsilon$ yields the following series of equations:

$$
\begin{gathered}
\bar{\varphi}_{0}\left(\vec{r}_{0}\right)=\frac{<\delta\left(\vec{r}, \vec{r}_{0}\right) \varphi_{0}(\vec{r})>}{<\varphi_{0}(\vec{r})>} \\
\bar{\varphi}_{1}\left(\vec{r}_{0}\right)=\frac{<\delta\left(\vec{r}, \vec{r}_{0}\right) \varphi_{1}(\vec{r})>-\bar{\varphi}_{0}<\varphi_{1}(\vec{r})>}{<\varphi_{0}>} \\
\bar{\varphi}_{2}\left(\vec{r}_{0}\right)=\frac{<\delta\left(\vec{r}, \vec{r}_{0}\right) \varphi_{2}(\vec{r})>-\bar{\varphi}_{0}<\varphi_{2}(\vec{r})>-\bar{\varphi}_{1}<\varphi_{1}(\vec{r})>}{<\varphi_{0}>} \\
\vdots \\
\bar{\varphi}_{n}\left(\vec{r}_{o}\right)=\frac{<\delta\left(\vec{r}, \vec{r}_{0}\right) \varphi_{n}(\vec{r})>-\bar{\varphi}_{0}<\varphi_{n}(\vec{r})>\cdots-\bar{\varphi}_{n-1}<\varphi_{1}(\vec{r})>}{<\varphi_{0}>}
\end{gathered}
$$

Equation (26) yields the expected result for the unperturbed flux while Eq. (27) may be used to obtain the first-order normalized scalar flux by rewriting it as

$$
\bar{\varphi}_{1}\left(\vec{r}_{0}\right)=\frac{<g\left(\vec{r}, \vec{r}_{0}\right), \varphi_{1}(\vec{r})>}{<\varphi_{0}(\vec{r})>}
$$

where

$$
g\left(\vec{r}, \vec{r}_{0}\right) \equiv \delta\left(\vec{x}-\vec{x}_{0}\right) \delta\left(E-E_{0}\right) \delta\left(\hat{\Omega}-\hat{\Omega}_{0}\right)-\bar{\varphi}_{0}\left(\vec{x}_{0}, E_{0}, \hat{\Omega}_{0}\right)
$$

We operate on Eq. (11) in an unnormalized flux form with $\left\langle\Psi_{0}^{*} \cdot>\right.$. The function, $\Psi_{0}^{*}$, is a yet-to-be defined adjoint function. After using the divergence theorem, vector identities, and a change of variable, we arrive at an equation that is very similar to Eq. (18) or

$$
<\varphi_{1} \Psi_{0}^{*} \hat{\Omega} \cdot \hat{n}>_{s}+<\varphi_{1}\left(H^{*}-\lambda_{0} F^{*}\right) \Psi_{0}^{*}>=\lambda_{1}<\Psi_{0}^{*} F \varphi_{0}>
$$

We define $\Psi_{o}^{*}$ as the solution to 


$$
\begin{gathered}
\left(H^{*}-\lambda_{0} F^{*}\right) \Psi_{0}^{*}=g \\
\Psi_{0}^{*}(x, E, \hat{\Omega})=\gamma_{0} \Psi_{0}^{*}\left(x, E, \hat{\Omega}_{r}\right), \vec{x} \in \partial \Re, \hat{\Omega} \cdot \hat{n}<0 .
\end{gathered}
$$

By using the unnormalized form of Eq. (15) in Eq. (32) we can rewrite this as

$$
<\gamma_{1} \Psi_{0}^{*}(\hat{\Omega}) \varphi_{0}\left(\hat{\Omega}_{r}\right) \hat{\Omega} \cdot \hat{n}>_{s, \hat{\Omega} \cdot \hat{n}<0}+<\varphi_{1}, g>=\lambda_{1}<\Psi_{0}^{*} F \varphi_{0}>
$$

Note that for $\Psi_{0}^{*}\left(\vec{x}, \vec{x}_{0}, E, E_{0}, \hat{\Omega}, \hat{\Omega}_{0}\right)$ to exist, the Fredholm alternative ${ }^{13}$ must be satisfied:

$$
<\varphi_{o} g>=0
$$

This expression holds because it can be rewritten as Eq. (26).

Since $\Psi_{0}^{*}$ is not unique, we define the following uniqueness condition to simplify Eq. (32).

$$
<\Psi_{0}^{*} F \varphi_{0}>=0
$$

Combine Eq. (37) with Eqs. (32) and (35) gives

$$
\bar{\varphi}_{1}\left(\vec{x}_{0}, E_{0}, \hat{\Omega}_{0}\right)=\frac{-<\gamma_{1} \Psi_{0}^{*}(\hat{\Omega}) \varphi_{0}\left(\hat{\Omega}_{r}\right) \hat{\Omega} \cdot \hat{n}>_{s, \hat{\Omega} \cdot \hat{n}<0}}{<\varphi_{0}(\vec{x})>}=-<\gamma_{1} \Psi_{0}^{*}(\hat{\Omega}) \bar{\varphi}_{0}\left(\hat{\Omega}_{r}\right) \hat{\Omega} \cdot \hat{n}>_{s, \hat{\Omega} \cdot \hat{n}<0}
$$

The above derivation can be expanded up to an arbitrary order, $n$, by following methods presented in Ref. 10. Operate on the higher-order expansion of the transport equation, Eq. (13), with $\left\langle\bar{\varphi}_{0}^{*} \cdot>\right.$ and use vector identities, the divergence theorem and a change of variables to get

$$
\begin{aligned}
& <\bar{\varphi}_{n} \bar{\varphi}_{0}^{*} \hat{\Omega} \cdot \hat{n}>_{s}+<\bar{\varphi}_{n}\left(H^{*}-\lambda_{0} F^{*}\right) \bar{\varphi}_{0}^{*}>= \\
& \lambda_{1}<\bar{\varphi}_{0}^{*} F \bar{\varphi}_{n-1}>+\lambda_{2}<\bar{\varphi}_{0}^{*} F \bar{\varphi}_{n-2}>\cdots+\lambda_{n}<\bar{\varphi}_{0}^{*} F \bar{\varphi}_{0}>
\end{aligned}, n>1
$$

We rearrange this equation and apply the definition of $\bar{\varphi}_{0}^{*}$ along with the $n^{\text {th }}$-order boundary condition expansion in Eq. (17) to get an equation for any higher-order eigenvalue term: 


$$
\lambda_{n}=\frac{\left\langle\gamma_{1} \bar{\varphi}_{0}^{*}(\hat{\Omega}) \bar{\varphi}_{n-1}\left(\hat{\Omega}_{r}\right) \hat{\Omega} \cdot \hat{n}>_{s, \hat{\Omega} \cdot \hat{n}<0}-\lambda_{1}<\bar{\varphi}_{0}^{*} F \bar{\varphi}_{n-1}>-\lambda_{2}<\bar{\varphi}_{0}^{*} F \bar{\varphi}_{n-2}>\cdots-\lambda_{n-1}<\bar{\varphi}_{0}^{*} F \bar{\varphi}_{1}>\right.}{<\bar{\varphi}_{0}^{*} F \bar{\varphi}_{0}>} .
$$

Deriving an expression for the flux starts with rewriting Eq. (29) as

$$
\bar{\varphi}_{n}\left(\vec{r}_{0}\right)=\frac{<g, \varphi_{n}(\vec{r})>-\bar{\varphi}_{1}\left(\vec{r}_{0}\right)<\varphi_{n-1}(\vec{r})>\cdots-\bar{\varphi}_{n-1}\left(\vec{r}_{0}\right)<\varphi_{1}(\vec{r})>}{<\varphi_{0}(\vec{r})>}
$$

We can derive an equation similar to Eq. (39) by operating on the unnormalized form of Eq. (13) with $<\Psi_{o}^{*} \cdot>$ and performing similar manipulations to get

$$
\begin{aligned}
& \left.<\varphi_{n} \Psi_{0}^{*} \hat{\Omega} \cdot \hat{n}\right\rangle_{s}+<\varphi_{n}\left(H^{*}-\lambda_{o} F^{*}\right) \Psi_{0}^{*}> \\
& =\lambda_{1}<\Psi_{0}^{*} F \varphi_{n-1}>+\lambda_{2}<\Psi_{0}^{*} F \varphi_{n-2}>\cdots+\lambda_{n}<\Psi_{0}^{*} F \varphi_{0}>
\end{aligned} .
$$

Use of Eq. (33) yields

$$
\begin{aligned}
& <g, \varphi_{n}>=\lambda_{1}<\Psi_{o}^{*} F \varphi_{n-1}>+\lambda_{2}<\Psi_{o}^{*} F \varphi_{n-2}>\cdots \\
& +\lambda_{n}<\Psi_{o}^{*} F \varphi_{o}>-<\gamma_{1} \Psi_{0}^{*}(\hat{\Omega}) \bar{\varphi}_{n-1}(\hat{\Omega} r) \hat{\Omega} \cdot \hat{n}>_{s, \hat{\Omega} \cdot \hat{n}<0}
\end{aligned} .
$$

We can write the integral terms on the right hand side in terms of normalized fluxes by operating on Eq. (29) with $\left\langle\Psi_{o}^{*} F \cdot>\right.$ to get

$$
<\Psi_{0}^{*} F \varphi_{k}>=<\Psi_{0}^{*} F \bar{\varphi}_{k}><\varphi_{0}>+<\Psi_{0}^{*} F \bar{\varphi}_{k-1}><\varphi_{1}>\cdots+<\Psi_{0}^{*} F \bar{\varphi}_{0}><\varphi_{k}>
$$

where $k$ varies from 1 to $n$-1. We substitute Eq. (43) into Eq. (41) and expand it with Eq. (44). Terms of $\left\langle\varphi_{k}\right\rangle$, where $k$ goes from 1 to $n-1$, drop out due to cancellation. Only the $\left\langle\varphi_{o}\right\rangle$ term remains to give a formula for the higher-order flux:

$$
\bar{\varphi}_{n}=\lambda_{1}<\Psi_{0}^{*} F \bar{\varphi}_{n-1}>+\lambda_{2}<\Psi_{0}^{*} F \bar{\varphi}_{n-2}>\cdots+\lambda_{n-1}<\Psi_{0}^{*} F \bar{\varphi}_{1}>-<\gamma_{1} \Psi_{0}^{*}(\hat{\Omega}) \bar{\varphi}_{n-1}\left(\hat{\Omega}_{r}\right) \hat{\Omega} \cdot \hat{n}>_{s, \hat{\Omega} \cdot \hat{n}<0}
$$

which can be proved by induction similar to the method presented in Ref. 10 . 


\section{NUMERICAL TECHNIQUES}

The adjoint, $\Psi_{0}^{*}$, does not have a straight forward numerical solution. The difficulty lies in the uniqueness condition presented in Eq. (37). This condition does not lend itself well to numerical manipulation. However, we can find a method to solve for the unique adjoint given an arbitrary particular solution, $\Psi_{p}^{*}$, to Eqs. (33) and (34).

The unique solution may be written as a particular solution plus a linear combination of the null space solution or

$$
\Psi_{0}^{*}\left(\vec{r}_{0}, \vec{r}\right)=\Psi_{p}^{*}\left(\vec{r}_{0}, \vec{r}\right)+\alpha\left(\vec{r}_{0}\right) \bar{\varphi}_{0}^{*}(\vec{r})
$$

Operating on Eq. (46) with the uniqueness condition in Eq. (37) yields

$$
<\Psi_{0}^{*} F \bar{\varphi}_{0}>_{\vec{r}}=0=<\Psi_{p}^{*} F \bar{\varphi}_{0}>_{\vec{r}}+\alpha<\bar{\varphi}_{0}^{*} F \bar{\varphi}_{0}>_{\vec{r}}
$$

which may be used to solve for $\alpha$. Once $\alpha$ is known, Eq. (46) may be used to give the unique adjoint solution.

The solution for $\Psi_{0}^{*}$ can be solved iteratively by solving Eqs. (33) and (34) for a given $\vec{r}_{0}$ using numerical methods similar to the solution of $\bar{\varphi}_{0}^{*}$ in Eqs. (21) and (22). After each iteration, the uniqueness condition is satisfied by solving Eq. (47) for $\alpha$ and adjusting the solution using Eq. (46). Repeat until the convergence criterion is met. An outer iteration over $\vec{r}_{0}$ will give the complete solution to $\Psi_{0}^{*}$. 
Numerically, the delta function may be hard to work with. One technique that works well is to use a nodal method and integrate Eq. (33) over a node. The integration of the delta function over a node is equal to 0 . It is equal to 1 only if $\vec{r}_{0}$ is equal to $\vec{r}$. This term may be then treated as an additional source term.

\section{EXAMPLE PROBLEMS}

To verify the formalism presented above, four examples are provided. For simplicity, the examples are all modeled as mono-energetic, one-dimensional slabs using discrete-ordinates (S-N) approximations. Unless otherwise noted, the example problems use S-4 with 100 spatial mesh points. Scattering and fission reactions are assumed to be isotropic. The first two examples use a simple slab with one material and two of different initial boundary conditions. The next example is a two-region (fuel/moderator) problem. The final example approximates a BWR fuel assembly with three materials.

\section{IV.A. Single Material Slab with Vacuum Boundary Conditions}

The first example is a simple slab of width 1 with $\sigma=v \sigma_{f}=1$ and $\sigma_{s}=0.5$. The surfaces are exposed to a vacuum boundary condition $\left(\gamma_{0}=0\right.$ in Eq. (6)). We perturb the right face boundary condition by changing $\varepsilon \gamma_{1}$ in Eq. (14) from 0.0 to 1.0. Table I shows the eigenvalue and the corresponding error as a function of albedo and perturbation order. Table II shows the \% RMS errors for the flux for the same albedoe. The errors in Tables I and II are defined as 


$$
\begin{gathered}
\% \text { error }=100\left(\frac{x_{\text {exact }}-x_{\text {predicted }}}{x_{\text {exact }}}\right) \text { and } \\
\% R M S=\sqrt{\frac{\sum_{i=1}^{N}\left(\frac{x_{\text {exact }}-x_{i}}{x_{\text {exact }}}\right)^{2}}{N}} .
\end{gathered}
$$

There are two points to note. The first is that first-order analysis is typically good enough up to $\varepsilon \gamma_{l}=0.5$. The higher-order terms become more important as the albedo is further increased. The second is that even at higher-orders for some of the mid-range albedos, the errors do not go away or even diminish past a certain point. This will be further discussed in the next section.

\section{IV.B. Single Material Slab with Specular Reflection Boundary Conditions}

The second example has the same material composition and dimension as the previous example.

The main difference is the starting (unperturbed) boundary condition has full specular reflection that is perturbed. The perturbation ranges from a vacuum boundary condition $\left(\varepsilon \gamma_{1}=-1.0\right)$ to double neutron reflection $\left(\varepsilon \gamma_{1}=1.0\right)$. Table III shows the eigenvalue computations for the higher-order terms and their respective errors. Table IV shows the \%RMS errors in the flux for the higher-order perturbation predictions.

The results of this example are consistent with what was seen in the previous example. We once again have higher-order terms with errors that are larger than a lower-order term. Also at the extreme perturbations, the higher-order terms become very important. 


\section{IV.C. Two Region (Fuel / Reflector) Slab}

The third example adds in an extra region to the idealized homogenous slab of the previous examples.. This example is modeled after a simple fuel and reflector slab example. The length of each region is 0.5. The fuel is made of the same material as given in Examples 1 and 2. The reflector is a purely scattering material with $\sigma=\sigma_{s}=1$. The fuel side has a specular reflection boundary condition imposed on it and the reflector has a vacuum boundary condition. The reflector's boundary condition is perturbed by $\varepsilon \gamma_{l}$ in Eq. (14). Table V shows the eigenvalues and their errors while Table VI shows the errors associated with the higher-order flux predictions.

The albedo is increased up to 1.75. At very large perturbations, the higher-order terms reduce the eigenvalue error from $-3981 \%$ down to $6.5 \%$ and the flux error from 105.3 to 0.38 . This shows that in cases where the perturbations may become large for a problem, higher-order theory is needed to improve the accuracy.

\section{IV.D. Three Region Fuel Assembly Slab}

The final example attempts to model a BWR fuel assembly in one dimension based on the work done in Ref. 14. The example consists of three regions, two fuel and one water. The setup is shown in Fig. 1 and the cross sections are defined in Table VII. 
The boundary condition at the external boundary of the water is perturbed. The fuel boundary condition is treated as a symmetry condition (full specular reflection). Therefore, an equivalent representation of this example is to see it as six regions with both water boundary conditions perturbed by the same amount. Table VIII shows the results of the higher-order eigenvalue perturbations while Table IX shows the errors in the flux.

Tables VIII and IX show much of the same information as previously seen in the other examples. One of the main differences is that the higher-order perturbation terms become important with small changes in the perturbation factor, $\varepsilon \gamma_{l}$. The range of the albedo changes is within realistic limits so this example shows us a practical case that definitely needs higher-order analysis to maintain accuracy.

The scalar flux is plotted in Fig. 2 for $\varepsilon \gamma_{1}=0.20$ in the water and part of fuel 2 region. This region was chosen since it shows a large enough perturbation that the results of a $5^{\text {th }}$ order analysis can be seen. Initially the zero ${ }^{\text {th }}$-order solution is basically flat. The $1^{\text {st }}$ order flux shows a large improvement in modeling the exact scalar flux. By $5^{\text {th }}$-order the perturbation estimate is close to the exact solution and could still use even higher-order to improve the accuracy if so desired. Plots for lower perturbations follow the same trend, but they are not as pronounced as given in Fig. 2 .

\section{DISCUSSION}


In the previous examples, it was noted that some of the higher-order solutions had errors the same as or higher than some lower-order terms. This is due to propagation of error in the perturbation terms. From examining Eqs. (40) and (45), one can see that each term is a function of all previous lower-order terms. So any error in the first-order terms is carried to the second-order terms. The first and second-order errors are thus carried to the third-order terms and so forth. The end result is the higher-order terms are overshadowed by the errors in the previous terms.

We verified that propagation of error was the cause of the errors observed by directly solving for the higher-order flux and eigenvalue terms in Eqs. (11) - (17). The direct solutions were more accurate than the solutions found by using the perturbation solutions from Eqs. (40) and (45). The direct solutions showed the errors consistently getting smaller for higher-order analysis.

While the direct solution should also suffer from propagation of error since each order is based on solutions of the pervious order, the direct solution seems to be able to go to orders very high in analysis before this happens. This is most likely due to the limited precision of the adjoint function, $\Psi_{0}^{*}$. The errors most likely arise from the way the numerical methods model the Dirac delta function found in Eq. (33).

\section{CLOSING REMARKS}

In this paper, we derived formalisms for the computation of higher-order flux and eigenvalue terms for a boundary condition perturbation. Numerical implementation for the solution to the 
adjoints was also discussed. Four numerical examples were presented and discussed as well as the errors associated with the errors associated with some of the higher-order solutions.

In this paper we have considered eigenvalue (criticality) problems only. It would be interesting to extend the method to fixed source problems in which a perturbation in the boundary condition of the system is of interest.

In reference 8 , an equivalence theory was developed to treat a perturbation in the external boundary of the system as a perturbation in the boundary condition of the system. This method was based on first-order perturbation theory. If the equivalence theory is extended to higher order then the method presented here can be used to determine the change in the solution due to a perturbation in the external boundary of the system with arbitrary accuracy.

Applications of the presented methods should take into consideration the high computational cost of computing the adjoint function, $\Psi_{0}^{*}$. One may solve for this equation by solving a fixed source problem once for each phase space point. Therefore applications should treat this as a precomputation that needs to be solved only once and the results stored for later computations.

As an example, the method presented herein may be applied to high-order homogenization methods as seen for the diffusion approximation in reference 11. This would allow for the correction of the homogenized cross sections for the effect of neutron leakage to any order of accuracy facilitating the development of efficient numerical transport methods for criticality analysis of large systems with heterogeneous coarse-mesh grids. 


\section{REFERENCES}

1. M. KOMATA, “A Generalized Perturbation Theory Applicable to Reactor Boundary Changes”, Nucl. Sci. Eng., 64, 811, (1977).

2. F. RAHNEMA and G. C. POMRANING, “On Linear Extrapolation Distances, Extrapolated Endpoints, and Eigenvalues", Nucl. Sci. Eng., 77, 438 (1981).

3. F. RAHNEMA, "Boundary Condition Perturbation Theory for Use in Spatial Homogenization Methods", Nucl. Sci. Eng., 102, 183 (1989).

4. H. R. KIM and N. Z. CHO, Ann. Nucl. Energy, 20, 11, 767 (1993).

5. F. RAHNEMA, "Perturbation Technique for the Reconstruction of Local Power and Flux Distribution in Nodal Methods", Topical Meeting on Advances in Reactor Physics: Reactor Physics Faces the 21st Century, Knoxville, TN, USA, April 11-15, 1994, II, 204, Am. Nucl. Soc. (1994).

6. F. RAHNEMA, “Boundary Condition Perturbations in Transport Theory”, Nucl. Sci. Eng., 124, 320 (1996). 
7. F. RAHNEMA, "Boundary Condition Perturbation Technique for Use in Spatial Homogenization Method in Transport Theory", Transport Theory \& Statistical Physics, 26, 575 (1997).

8. F. RAHNEMA and P. RAVETTO, "On the Equivalence of Boundary and Boundary Condition Perturbations in Transport Theory and Its Diffusion Approximation”, Nucl. Sci. Eng., 128, 209, (1998).

9. M. S. MCKINLEY and F. RAHNEMA, "A Second-Order Accurate Eigenvalue Expression for Boundary Condition Perturbation", International Conference on Mathematics and Computation, Reactor Physics and Environmental Analysis in Nuclear Applications, Madrid, Spain, Sept. 27-30, Am. Nucl. Soc. (1999).

10. M. S. MCKINLEY and F. RAHNEMA, "Higher-Order Boundary Condition Perturbation Theory for the Diffusion Approximation", Nucl. Sci. Eng., 136, 15, (2000).

11. F. RAHNEMA and M. S. MCKINLEY, "High-Order Cross Section Homogenization Method, " Ann Nucl. Energy, accepted (April 2001).

12. K. S. SMITH, “Assembly Homogenization Techniques for Light Water Reactor Analysis," Prog. Nucl. Energy, 17, 303 (1986).

13. R. COURANT and D. HILBERT, Methods of Mathematical Physics, Vol I, p. 116, Wiley Interscience Publishers, New York, (1953). 
14. F. RAHNEMA and E. M. NICHITA, "Leakage Corrected Spatial (Assembly)

Homogenization Techniques”, Ann. Nucl. Energy, 20, 477 (1997). 
TABLE I ${ }^{\mathrm{a}}$

Comparison of Higher-Order Eigenvalue Terms for Example 1

\begin{tabular}{|c|c|c|c|c|c|c|c|}
\hline$\varepsilon \gamma_{l}$ & Exact & $0^{\text {th }}-$ Order & $1^{\text {st }}-$ Order & $2^{\text {nd- }}$ Order & $3^{\text {rd }}-$ Order & $4^{\text {th }}-$ Order & $5^{\text {th }}-$ Order \\
\hline 0.01 & 1.1812 & $1.1857(-0.4)$ & $1.1812(0.0)$ & $1.1812(0.0)$ & $1.1812(0.0)$ & $1.1812(0.0)$ & $1.1812(0.0)$ \\
\hline 0.05 & 1.1632 & $1.1857(-1.9)$ & $1.1630(0.0)$ & $1.1631(0.0)$ & $1.1631(0.0)$ & $1.1631(0.0)$ & $1.1631(0.0)$ \\
\hline 0.10 & 1.1409 & $1.1857(-3.9)$ & $1.1403(0.1)$ & $1.1407(0.0)$ & $1.1408(0.0)$ & $1.1408(0.0)$ & $1.1408(0.0)$ \\
\hline 0.50 & 0.9721 & $1.1857(-22.0)$ & $0.9588(1.4)$ & $0.9692(0.3)$ & $0.9727(-0.1)$ & $0.9719(0.0)$ & $0.9719(0.0)$ \\
\hline 1.00 & 0.7896 & $1.1857(-50.1)$ & $0.7318(7.3)$ & $0.7734(2.1)$ & $0.8014(-1.5)$ & $0.7894(0.0)$ & $0.7881(0.2)$ \\
\hline
\end{tabular}

${ }^{\mathrm{a}}$ The numbers in parenthesis are percent errors from the exact solution 
TABLE II

Comparison of Higher-Order Flux Errors for Example 1

\begin{tabular}{|c|c|c|c|c|c|c|}
\hline$\varepsilon \gamma_{1}$ & $\begin{array}{c}0^{\text {th }} \text {-Order } \\
\% \text { RMS }\end{array}$ & $\begin{array}{c}1^{\text {st }}-\text { Order } \\
\% R M S\end{array}$ & $\begin{array}{c}2^{\text {nd }} \text {-Order } \\
\% R M S\end{array}$ & $\begin{array}{c}3^{\text {rd }} \text {-Order } \\
\% R M S\end{array}$ & $\begin{array}{c}4^{\text {th }} \text {-Order } \\
\% R M S\end{array}$ & $\begin{array}{c}5^{\text {th }} \text {-Order } \\
\% R M S\end{array}$ \\
\hline 0.01 & 5.12 & 0.01 & 0.01 & 0.01 & 0.01 & 0.01 \\
\hline 0.05 & 11.60 & 0.11 & 0.02 & 0.02 & 0.02 & 0.02 \\
\hline 0.10 & 15.79 & 0.30 & 0.03 & 0.03 & 0.03 & 0.03 \\
\hline 0.50 & 32.51 & 4.24 & 0.32 & 0.17 & 0.04 & 0.05 \\
\hline 1.00 & 50.10 & 15.94 & 2.43 & 1.66 & 0.71 & 0.18 \\
\hline
\end{tabular}


TABLE III ${ }^{\mathrm{a}}$

Comparison of Higher-Order Eigenvalue Terms for Example 2

\begin{tabular}{|c|c|c|c|c|c|c|c|}
\hline$\varepsilon \gamma_{1}$ & Exact & $0^{\text {th }}-$ Order & $1^{\text {st }}-$ Order & $2^{\text {nd- }}$ Order & $3^{\text {rd }}-$ Order & $4^{\text {th }}-$ Order & $5^{\text {th }}-$ Order \\
\hline-1.00 & 0.7896 & $0.5000(36.7)$ & $0.7606(3.7)$ & $0.7957(-0.8)$ & $0.7942(-0.6)$ & $0.7913(-0.2)$ & $0.7905(-0.1)$ \\
\hline-0.75 & 0.7130 & $0.5000(29.9)$ & $0.6955(2.5)$ & $0.7152(-0.3)$ & $0.7146(-0.2)$ & $0.7137(-0.1)$ & $0.7135(-0.1)$ \\
\hline-0.50 & 0.6385 & $0.5000(21.7)$ & $0.6303(1.3)$ & $0.6391(-0.1)$ & $0.6389(-0.1)$ & $0.6387(0.0)$ & $0.6387(0.0)$ \\
\hline-0.10 & 0.5264 & $0.5000(5.0)$ & $0.5261(0.1)$ & $0.5264(0.0)$ & $0.5264(0.0)$ & $0.5264(0.0)$ & $0.5264(0.0)$ \\
\hline-0.05 & 0.5131 & $0.5000(2.6)$ & $0.5130(0.0)$ & $0.5131(0.0)$ & $0.5131(0.0)$ & $0.5131(0.0)$ & $0.5131(0.0)$ \\
\hline-0.01 & 0.5026 & $0.5000(0.5)$ & $0.5026(0.0)$ & $0.5026(0.0)$ & $0.5026(0.0)$ & $0.5026(0.0)$ & $0.5026(0.0)$ \\
\hline 0.01 & 0.4974 & $0.5000(-0.5)$ & $0.4974(0.0)$ & $0.4974(0.0)$ & $0.4974(0.0)$ & $0.4974(0.0)$ & $0.4974(0.0)$ \\
\hline 0.05 & 0.4871 & $0.5000(-2.7)$ & $0.4870(0.0)$ & $0.4871(0.0)$ & $0.4871(0.0)$ & $0.4871(0.0)$ & $0.4871(0.0)$ \\
\hline 0.10 & 0.4743 & $0.5000(-5.4)$ & $0.4739(-0.1)$ & $0.4743(0.0)$ & $0.4743(0.0)$ & $0.4743(0.0)$ & $0.4743(0.0)$ \\
\hline 0.50 & 0.3784 & $0.5000(-32.1)$ & $0.3697(2.3)$ & $0.3785(0.0)$ & $0.3787(-0.1)$ & $0.3785(0.0)$ & $0.3785(0.0)$ \\
\hline 0.75 & 0.3240 & $0.5000(-54.3)$ & $0.3045(6.0)$ & $0.3243(-0.1)$ & $0.3249(-0.3)$ & $0.3240(0.0)$ & $0.3242(-0.1)$ \\
\hline 1.00 & 0.2736 & $0.5000(-82.7)$ & $0.2394(12.5)$ & $0.2745(-0.3)$ & $0.2760(-0.9)$ & $0.2732(0.2)$ & $0.2740(-0.1)$ \\
\hline
\end{tabular}

${ }^{a}$ the number in parenthesis are percent errors from the exact solution 
TABLE IV

Comparison of Higher-Order Flux Errors for Example 2

\begin{tabular}{|c|c|c|c|c|c|c|}
\hline$\varepsilon \gamma_{1}$ & $\begin{array}{c}0^{\text {th }} \text {-Order } \\
\% \text { RMS }\end{array}$ & $\begin{array}{c}1^{\text {st }} \text {-Order } \\
\% R M S\end{array}$ & $\begin{array}{c}2^{\text {nd }} \text {-Order } \\
\% \text { RMS }\end{array}$ & $\begin{array}{c}3^{\text {rd }} \text {-Order } \\
\% \text { RMS }\end{array}$ & $\begin{array}{c}4^{\text {th }} \text {-Order } \\
\% R M S\end{array}$ & $\begin{array}{c}5^{\text {th }} \text {-Order } \\
\% R M S\end{array}$ \\
\hline-1.00 & 1413.41 & 182.07 & 16.27 & 16.19 & 8.26 & 4.63 \\
\hline-0.75 & 65.89 & 8.34 & 0.65 & 0.43 & 0.25 & 0.18 \\
\hline-0.50 & 27.51 & 2.57 & 0.14 & 0.07 & 0.05 & 0.04 \\
\hline-0.10 & 3.75 & 0.08 & 0.00 & 0.00 & 0.00 & 0.00 \\
\hline-0.05 & 1.81 & 0.02 & 0.00 & 0.00 & 0.00 & 0.00 \\
\hline-0.01 & 0.35 & 0.00 & 0.00 & 0.00 & 0.00 & 0.00 \\
\hline 0.01 & 0.35 & 0.00 & 0.00 & 0.00 & 0.00 & 0.00 \\
\hline 0.05 & 1.71 & 0.02 & 0.00 & 0.00 & 0.00 & 0.00 \\
\hline 0.10 & 3.33 & 0.07 & 0.00 & 0.00 & 0.00 & 0.00 \\
\hline 0.50 & 14.18 & 1.65 & 0.15 & 0.02 & 0.01 & 0.01 \\
\hline 0.75 & 19.93 & 3.59 & 0.51 & 0.07 & 0.01 & 0.03 \\
\hline 1.00 & 25.35 & 6.24 & 1.20 & 0.17 & 0.07 & 0.07 \\
\hline
\end{tabular}


TABLE $\mathrm{V}^{\mathrm{a}}$

Comparison of Higher-Order Eigenvalue Terms for Example 3

\begin{tabular}{|c|c|c|c|c|c|c|c|}
\hline$\varepsilon \gamma_{1}$ & Exact & $0^{\text {th }}-$ Order & $1^{\text {st }}-$ Order & $2^{\text {nd- }}$ Order & $3^{\text {rd }}-$ Order & $4^{\text {th }}-$ Order & $5^{\text {th }}-$ Order \\
\hline 0.01 & 0.9151 & $0.9185(-0.4)$ & $0.9151(0.0)$ & $0.9151(0.0)$ & $0.9151(0.0)$ & $0.9151(0.0)$ & $0.9151(0.0)$ \\
\hline 0.05 & 0.9015 & $0.9185(-1.9)$ & $0.9016(0.0)$ & $0.9014(0.0)$ & $0.9014(0.0)$ & $0.9014(0.0)$ & $0.9014(0.0)$ \\
\hline 0.10 & 0.8842 & $0.9185(-3.9)$ & $0.8846(0.0)$ & $0.8840(0.0)$ & $0.8839(0.0)$ & $0.8839(0.0)$ & $0.8839(0.0)$ \\
\hline 0.15 & 0.8666 & $0.9185(-6.0)$ & $0.8677(-0.1)$ & $0.8662(0.0)$ & $0.8662(0.1)$ & $0.8661(0.1)$ & $0.8661(0.1)$ \\
\hline 0.30 & 0.8114 & $0.9185(-13.2)$ & $0.8168(-0.7)$ & $0.8109(0.1)$ & $0.8106(0.1)$ & $0.8106(0.1)$ & $0.8106(0.1)$ \\
\hline 0.50 & 0.7322 & $0.9185(-25.4)$ & $0.7491(-2.3)$ & $0.7326(-0.1)$ & $0.7310(0.2)$ & $0.7309(0.2)$ & $0.7308(0.2)$ \\
\hline 0.75 & 0.6228 & $0.9185(-47.5)$ & $0.6644(-6.7)$ & $0.6274(-0.7)$ & $0.6219(0.1)$ & $0.6211(0.3)$ & $0.6210(0.3)$ \\
\hline 1.00 & 0.5000 & $0.9185(-83.7)$ & $0.5796(-15.9)$ & $0.5139(-2.8)$ & $0.5009(-0.2)$ & $0.4984(0.3)$ & $0.4980(0.4)$ \\
\hline 1.25 & 0.3612 & $0.9185(-154)$ & $0.4949(-37.0)$ & $0.3923(-8.6)$ & $0.3668(-1.5)$ & $0.3607(0.1)$ & $0.3594(0.5)$ \\
\hline 1.50 & 0.2034 & $0.9185(-352)$ & $0.4102(-102)$ & $0.2624(-29.0)$ & $0.2183(-7.3)$ & $0.2058(-1.2)$ & $0.2025(0.4)$ \\
\hline 1.75 & 0.0225 & $0.9185(-3981)$ & $0.3255(-1346)$ & $0.1243(-452)$ & $0.0543(-141)$ & $0.0311(-38.2)$ & $0.0240(-6.5)$ \\
\hline
\end{tabular}

${ }^{a}$ the number in parenthesis are percent errors from the exact solution 
TABLE VI

Comparison of Higher-Order Flux Errors for Example 3

\begin{tabular}{|c|c|c|c|c|c|c|}
\hline$\varepsilon \gamma_{1}$ & $\begin{array}{c}0^{\text {th }}-\text { Order } \\
\% R M S\end{array}$ & $\begin{array}{c}1^{\text {st }}-\text { Order } \\
\% R M S\end{array}$ & $\begin{array}{c}2^{\text {nd }} \text {-Order } \\
\% R M S\end{array}$ & $\begin{array}{c}3^{\text {rd }} \text {-Order } \\
\% R M S\end{array}$ & $\begin{array}{c}4^{\text {th }} \text {-Order } \\
\% R M S\end{array}$ & $\begin{array}{c}5^{\text {th }} \text {-Order } \\
\% R M S\end{array}$ \\
\hline 0.01 & 7.38 & 0.03 & 0.03 & 0.03 & 0.03 & 0.03 \\
\hline 0.05 & 15.52 & 0.08 & 0.07 & 0.07 & 0.07 & 0.07 \\
\hline 0.10 & 20.55 & 0.14 & 0.09 & 0.09 & 0.09 & 0.09 \\
\hline 0.15 & 23.96 & 0.21 & 0.10 & 0.10 & 0.10 & 0.10 \\
\hline 0.30 & 30.67 & 0.52 & 0.11 & 0.10 & 0.10 & 0.10 \\
\hline 0.50 & 36.74 & 1.03 & 0.12 & 0.09 & 0.09 & 0.09 \\
\hline 0.75 & 43.45 & 1.78 & 0.16 & 0.06 & 0.05 & 0.05 \\
\hline 1.00 & 51.21 & 2.66 & 0.27 & 0.03 & 0.00 & 0.00 \\
\hline 1.25 & 61.87 & 3.71 & 0.50 & 0.04 & 0.07 & 0.08 \\
\hline 1.50 & 78.11 & 5.06 & 0.93 & 0.10 & 0.16 & 0.19 \\
\hline 1.75 & 105.30 & 7.04 & 1.80 & 0.25 & 0.31 & 0.38 \\
\hline
\end{tabular}




\section{TABLE VII}

Cross Section Data for Each Material in Example 4

\begin{tabular}{|c|c|c|c|c|}
\hline Material & $\sigma_{\mathrm{t}}$ & $\sigma_{\mathrm{s}}{ }^{\mathrm{A}}$ & $\sigma_{\mathrm{a}}$ & $v \sigma_{\mathrm{f}}$ \\
\hline Fuel 1 & 0.2762 & 0.2478 & 0.0284 & 0.0416 \\
\hline Fuel 2 & 0.2762 & 0.251 & 0.0252 & 0.034 \\
\hline Water & 3.45 & 3.45 & 0 & 0 \\
\hline
\end{tabular}

${ }^{\mathrm{A}}$ Isotropic scattering is assumed. 
TABLE VIII ${ }^{\mathrm{a}}$

Comparison of Higher-Order Eigenvalue Terms for Example 4

\begin{tabular}{|c|c|c|c|c|c|c|c|}
\hline$\varepsilon \gamma_{1}$ & Exact & $0^{\text {th }}-$ Order & $1^{\text {st }}-$ Order & $2^{\text {nd- }}$ Order & $3^{\text {rd }}-$ Order & $4^{\text {th }}-$ Order & $5^{\text {th }}-$ Order \\
\hline-0.20 & 0.8358 & $0.7089(15.2)$ & $0.9199(-10.1)$ & $0.7833(6.3)$ & $0.8729(-4.4)$ & $0.8144(2.6)$ & $0.8524(-2.0)$ \\
\hline-0.15 & 0.8146 & $0.7089(13.0)$ & $0.8672(-6.5)$ & $0.7903(3.0)$ & $0.8281(-1.7)$ & $0.8096(0.6)$ & $0.8186(-0.5)$ \\
\hline-0.10 & 0.7881 & $0.7089(10.1)$ & $0.8144(-3.3)$ & $0.7802(1.0)$ & $0.7914(-0.4)$ & $0.7878(0.0)$ & $0.7890(-0.1)$ \\
\hline-0.05 & 0.7541 & $0.7089(6.0)$ & $0.7616(-1.0)$ & $0.7531(0.1)$ & $0.7545(0.0)$ & $0.7543(0.0)$ & $0.7543(0.0)$ \\
\hline-0.01 & 0.7191 & $0.7089(1.4)$ & $0.7194(0.0)$ & $0.7191(0.0)$ & $0.7191(0.0)$ & $0.7191(0.0)$ & $0.7191(0.0)$ \\
\hline 0.01 & 0.6980 & $0.7089(-1.6)$ & $0.6983(-0.1)$ & $0.6980(0.0)$ & $0.6980(0.0)$ & $0.6980(0.0)$ & $0.6980(0.0)$ \\
\hline 0.05 & 0.6456 & $0.7089(-9.8)$ & $0.6561(-1.6)$ & $0.6476(-0.3)$ & $0.6462(-0.1)$ & $0.6459(-0.1)$ & $0.6459(0.0)$ \\
\hline 0.10 & 0.5510 & $0.7089(-28.7)$ & $0.6033(-9.5)$ & $0.5692(-3.3)$ & $0.5580(-1.3)$ & $0.5543(-0.6)$ & $0.5531(-0.4)$ \\
\hline 0.15 & 0.3943 & $0.7089(-79.8)$ & $0.5506(-39.6)$ & $0.4737(-20.1)$ & $0.4359(-10.5)$ & $0.4173(-5.8)$ & $0.4083(-3.5)$ \\
\hline 0.20 & 0.0880 & $0.7089(-705)$ & $0.4978(-466)$ & $0.3611(-310)$ & $0.2715(-209)$ & $0.2130(-142)$ & $0.1749(-98.7)$ \\
\hline
\end{tabular}

\footnotetext{
${ }^{a}$ the number in parenthesis are percent errors from the exact solution
} 
TABLE IX

Comparison of Higher-Order Flux Errors for Example 4

\begin{tabular}{|c|c|c|c|c|c|c|}
\hline$\varepsilon \gamma_{I}$ & $\begin{array}{c}0^{\text {th }}-\text { Order } \\
\% R M S\end{array}$ & $\begin{array}{c}1^{\text {st }}-\text { Order } \\
\% R M S\end{array}$ & $\begin{array}{c}2^{\text {nd }}-\text { Order } \\
\% R M S\end{array}$ & $\begin{array}{c}3^{\text {rd }}-\text { Order } \\
\% R M S\end{array}$ & $\begin{array}{c}4^{\text {th }}-\text { Order } \\
\% R M S\end{array}$ & $\begin{array}{c}5^{\text {th }}-\text { Order } \\
\% R M S\end{array}$ \\
\hline-0.20 & 17.28 & 10.20 & 5.84 & 3.46 & 1.69 & 1.22 \\
\hline-0.15 & 13.06 & 5.78 & 2.33 & 1.15 & 0.34 & 0.29 \\
\hline-0.10 & 8.85 & 2.61 & 0.68 & 0.26 & 0.01 & 0.07 \\
\hline-0.05 & 4.53 & 0.67 & 0.08 & 0.03 & 0.01 & 0.02 \\
\hline-0.01 & 0.93 & 0.03 & 0.00 & 0.00 & 0.00 & 0.00 \\
\hline 0.01 & 0.95 & 0.03 & 0.00 & 0.00 & 0.00 & 0.00 \\
\hline 0.05 & 4.98 & 0.73 & 0.13 & 0.04 & 0.03 & 0.03 \\
\hline 0.10 & 10.87 & 3.18 & 1.00 & 0.37 & 0.20 & 0.15 \\
\hline 0.15 & 18.84 & 8.23 & 3.72 & 1.80 & 0.99 & 0.65 \\
\hline 0.20 & 32.85 & 18.97 & 11.13 & 6.71 & 4.23 & 2.86 \\
\hline
\end{tabular}




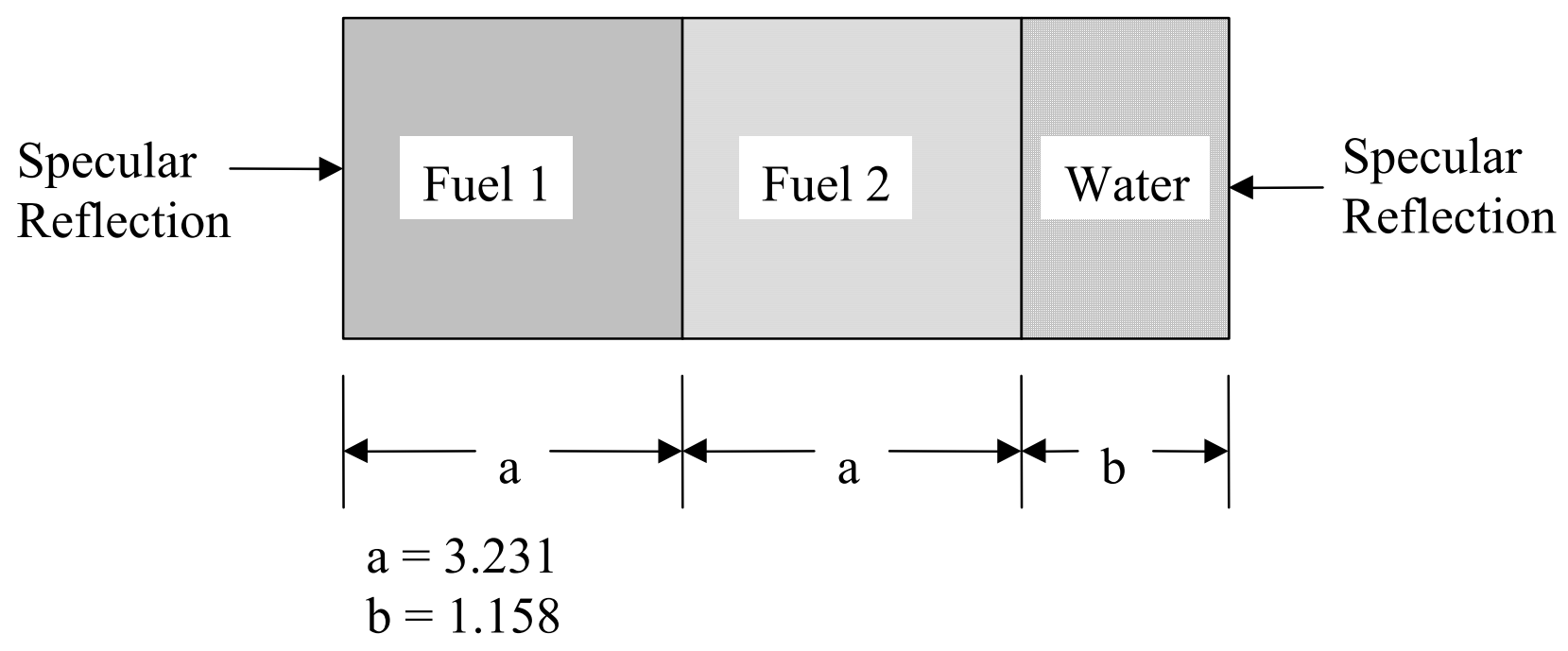




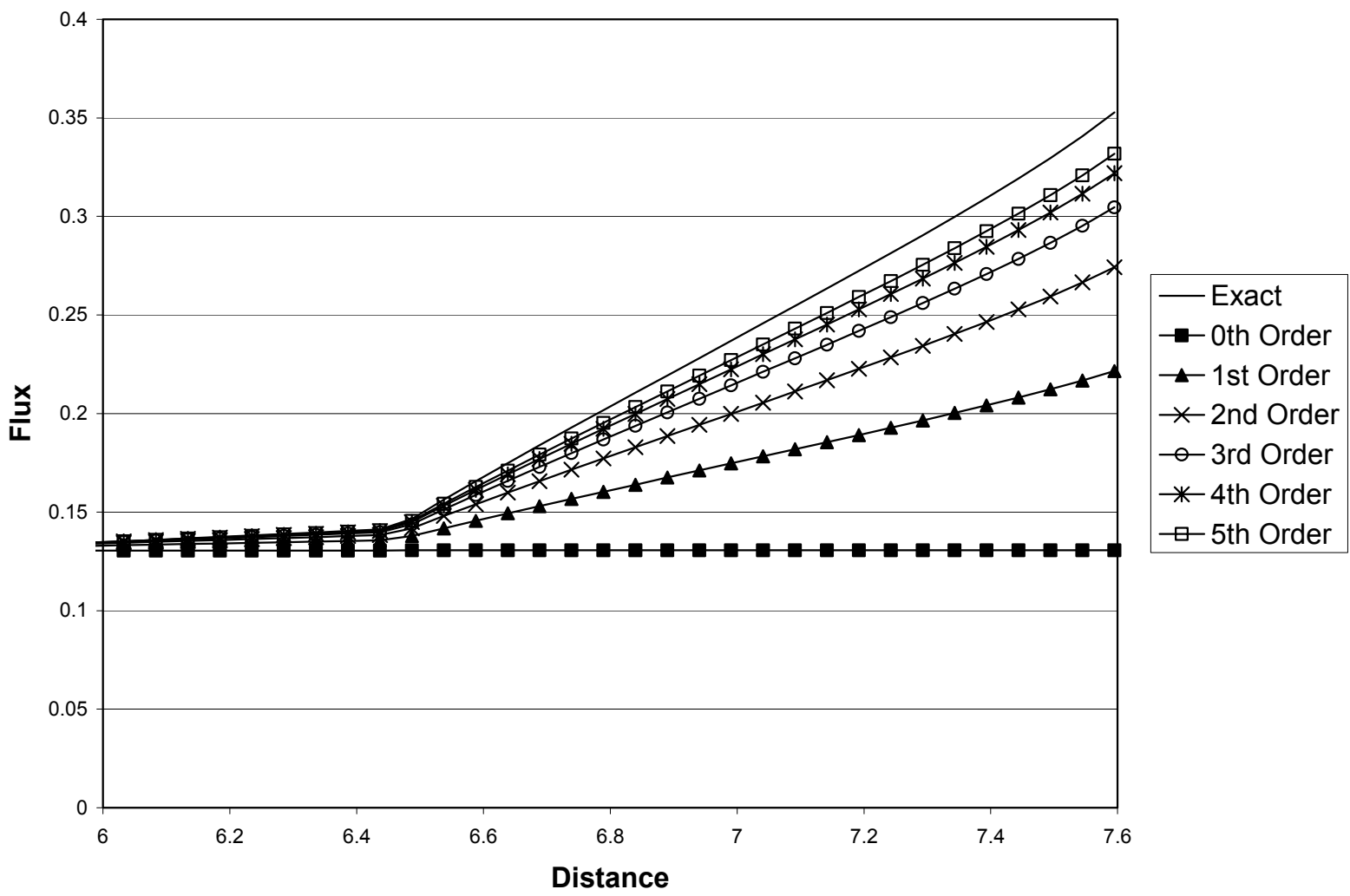


Fig. 1. Material Layout for Example 4

Fig. 2. Scalar Flux Plot for $\varepsilon \gamma_{l}=0.20$ for Example 4 